\title{
北京平谷上宅遗址骨柄石刀刀的微痕分析：来自 环境扫描电镜观察的证据
}

\author{
崔天兴 ${ }^{(1 *}$, 杨琴 $^{(1)}$, 郁金城 ${ }^{(2)}$, 周双林 ${ }^{(1)}$, 赵朝洪 ${ }^{(1)}$ \\ (1) 北京大学考古文博学院, 北京 100871; \\ (2) 北京市文物研究所, 北京 100009 \\ *E-mail: tianxingcui@163.com
}

收稿日期: 2009-05-15; 接受日期: 2010-05-30

国家社会科学基金项目(批准号: 08BKJ002 和 07CKG001)、教育部人文社科重点研究基地重大项目(批准号: 05JJD78002)、北京市哲学社 会科学“十一五”规划项目(编号: 06Aals002)和“东钱湖石刻病害调查与保护对策研究”项目资助

\begin{abstract}
摘要一般认为, 新石器时代遗址出土的骨柄石刀刀是一种狩猎采集经济工具,但近年 来不断受到学者的质疑. 本文使用环境扫描电镜对距今 7000 年前北京平谷上宅遗址出土 的一件骨柄石刀刀进行观察, 并对细石叶的器表残留物进行了分析. 环境扫描电镜的观 察和 $\mathrm{X}$ 射线能谱分析表明，骨柄石刃刀刃部残留有磷、钙、碳、氧等物质成分，为我们认 定其用途提供了直接证据, 表明其当时可能用来加工肉类. 分析还发现石刃背缘部位覆 盖有胶层并含有均匀分布的钡盐、锌盐等. 这一发现对重新认识史前居民制胶工艺提供了 新的线索, 但尚需要该遗址以及其他遗址的材料进一步支持.
\end{abstract}

关键词

上宅文化

骨柄石刃刀

微痕分析

环境扫描电镜

$\mathrm{X}$ 射线能谱分析
细石叶是旧石器时代晚期出现一种采用压制或 者间接打击的工艺技术而产生的一种长宽之比基本 大于 $2: 1$ 的标准化石制品, 它们是作为装备骨、木等 复合工具的石刃而专门制作的 ${ }^{[1 \sim 4]}$, 其出现是和更新 世末期的全球性气候变化中人类大迁移有密切关系 ${ }^{[5]}$. 考古出土的以细石叶为刃缘的复合工具即骨柄石 刃刀, 一般认为是狩猎采集的工具. 近年来随着农业 起源研究的进展, 考古学家根据实验考古学和微痕 分析对细石叶工具进行了重新的思考和定位, 认为 细石叶工具尤其是骨柄石刃刀不但和狩猎相关, 而 且还介入了植物驯化的过程 ${ }^{[6 ~ 11]}$. 通过微痕观察, Unger-Hamilton ${ }^{[7]}$ 认为 “这些石刀 (石叶工具) 的光泽提 供了他们在 (收割) 植物方面的清楚的使用证据”. $\mathrm{Lu}^{[8 \sim 10]}$ 对山西旧石器时代晚期的下川遗址群细石叶
进行微痕分析后根据光泽类型指出, 下川遗址已存 在收割禾本科植物的实践. 王小庆 ${ }^{[11]}$ 分辨出赵宝沟 遗址出土细石叶工具的收割谷物 (A 型)和处理肉类、 兽皮(E1，E2 和 D 型)的光泽，从而认为“赵宝沟文化 的经济形态已经出现了农业生产的迹象”. 这些新的 认识多由考古标本与同类实验考古学标本上的微痕 和光泽的比较获得, 无直接证据揭示细石叶工具具 体的用途.

20 世纪 60 年代末期, 考古学家尝试运用普通扫 描电镜对考古标本进行观察分析 ${ }^{[12]}$. 受扫描电镜技 术在高真空模式下对样品导电性要求的限制, 对导 电性能不好或者不导电的考古样品, 有两种处理方 式：1）样品首先通过喷金或喷碳进行表面导电性处 理, 然后再进行观察. 主要应用于骨质微痕、植硅石

引用格式: Cui T X, Yang Q, Yu J C, et al. Microwear analysis of a bone-handled microblade excavated at Shangzhai Site in Beijing: Evidences from ESEM. Sci China Earth Sci, 2010, doi: 10.1007/s11430-010-4019-7 
鉴定研究 ${ }^{[13 ~ 15]}$ 、颜料分析 ${ }^{[16,17]}$ 等方面, 然而这会给考 古样品带来不可逆的污染或损害. 2) 使用合成复型 材料作为考古样品表面的替代品, 镀金或喷碳后进 行电镜观察, 主要应用于石器微痕分析或者较大样 品的局部观察分析等方面 ${ }^{[18,19]}$. 这种方法可以获得 超高倍率的二次电子图像, 但使扫描电镜对样品定 性定量分析手段无用武之地, 使得其与高倍法(金相 等光学显微镜) 相比, 对有关问题的解决并没有获得 质的突破和进展.

国内扫描电镜在考古学上的应用开始于 20 世纪 80 年代对古尸的形貌观察、寄生虫研究等方面 ${ }^{[20]}$, 发 展较为缓慢. 20 世纪 90 年代中期以来, 扫描电镜的

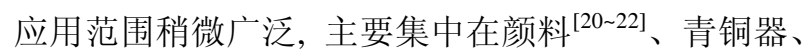
铁器、陶瓷器的研究 ${ }^{[23]}$ 、石器微痕分析 ${ }^{[19]}$ 、玉器 ${ }^{[24]}$ 等方面. 但是这些研究多为文物的保护、科学分析等 方面, 利用 SEM 进行考古样品的深度信息发掘还有 很大的不足. 也有学者应用常规扫描电镜对石制品 进行微痕分析, 但结果与金相显微镜相比, 并无突破 的进展 ${ }^{[19]}$.

20 世纪 90 年代以后, 环境扫描电镜(ESEM)出现. 与常规扫描电镜相比, 环境扫描电镜可以对各种固 体和液体样品进行形态观察和元素定性定量分析. 考古样品既不需要脱水, 也不必进行导电处理, 可在 自然的状态下直接观察二次电子图像 (SEI), 提供样 品的微观形貌结构, 并利用背散射电子(BEI )配合能 谱仪(EDS)分析元素成分百分比, 所以其在考古学研 究方面具有很广阔的应用前景. 在此之后, 利用 ESEM 对导电性能不好或者不导电的样品直接观察 开始出现, 但直到现在, 使用普通扫描电镜对样品进 行导电预处理后再观察的情况依然比较普遍 ${ }^{[23]}$.
ESEM 在考古学中的应用并没有得到广泛的推广.

本文在没有对考古样品进行导电处理的情况下， 通过使用 ESEM 对北京平谷上宅遗址出土的一件骨 柄石刀刀在自然状态进行了直接的观察, 并对其表 面残留物进行了 $\mathrm{X}$ 射线能谱分析, 为了解该器物的 功能提供了直接的证据, 也对 ESEM 进行考古样品 的分析进行了探索.

\section{1 研究材料与方法}

上宅遗址发现于 1984 年, 位于北京市平谷区城 东北 $17 \mathrm{~km}$ 金海湖镇上宅村西北边的一块台地上, 南 临泃河, 北靠燕山. 1984 1987 年进行发掘, 总发掘 面积约 $2860 \mathrm{~m}^{2}$. 整个遗址共分为 8 层, 其中 (1), (2)为 晚期文化层; (3) 8) 是新石器时代的文化地层, 其中 (4) (7) 是以上宅文化为内涵的新石器时代文化层 ${ }^{[25]}$; 具体年代如表 1 所示.

北京大学 ${ }^{14} \mathrm{C}$ 年代实验室对采自文化层的木炭 样品进行了年代测定, 共测得 4 个数据 ${ }^{[25,26]}$, 但均未 进行树轮校正和同位素分馏校正, 树轮校正以后如 表 1 .

上宅遗址出土陶器共 1000 余件, 石器约 2000 余 件; 石器大多为打制和磨制的大型石器, 也有一定数 量以骨柄石刃刀为代表的复合工具.

本次实验选择编号为 T0607(5):40, 41(图 1)的骨 柄石刃刀进行环境扫描电镜分析. 出土时刃和柄部 脱离. 残骨柄呈柳叶形, 骨柄从中部断开, 残长 74.2 $\mathrm{mm}$, 骨柄厚 9.2 14.4 mm, 宽 6.1 6.8 $\mathrm{mm}$, 重 $6.2 \mathrm{~g}$; 背脊光滑圆针, 凹槽口外宽为 $5.2 \mathrm{~mm}$, 内宽 $4.1 \mathrm{~mm}$, 凹槽剖面呈“V”字形, 刀头处稍浅, 深 $3.2 \mathrm{~mm}$, 最深

表 1 上宅遗址 ${ }^{14} \mathrm{C}$ 年代数据表 $(\mathrm{OxCal} \mathrm{3.10})^{\mathrm{a})}$

\begin{tabular}{|c|c|c|c|c|c|}
\hline 实验室编号 & 样品层位 & 样品 & ${ }^{14} \mathrm{C}(5730)$ 年代 & ${ }^{14} \mathrm{C}(5568)$ 年代 & 校正年代(BC) \\
\hline BK85077 & T05088)层 & 木炭 & $6580 \pm 120$ & $6395 \pm 120$ & $\begin{array}{c}5480 \sim 5290(59.5 \%) \\
5270 \sim 5220(8.7 \%) \\
5650 \sim 5050(95.4 \%)\end{array}$ \\
\hline BK85079 & T0309(7)层 & 木炭 & $6540 \pm 100$ & $6356 \pm 100$ & $\begin{array}{c}5470 \sim 5400(20.7 \%) \\
5390 \sim 5290(35.6 \%) \\
5270 \sim 5220(12.0 \%) \\
5510 \sim 5190(85.5 \%) \\
5180 \sim 5050(9.9 \%)\end{array}$ \\
\hline BK84019 & T1(5)层 & 木炭 & $6000 \pm 105$ & $5831 \pm 105$ & $\begin{array}{l}4800 \sim 4540(68.2 \%) \\
4950 \sim 4450(95.4 \%)\end{array}$ \\
\hline BK84109 & T0706(5)层 & 木炭 & $6340 \pm 200$ & $6161 \pm 200$ & $\begin{array}{l}5320 \sim 4840(68.2 \%) \\
5500 \sim 4600(95.4 \%)\end{array}$ \\
\hline
\end{tabular}

a) 据文献[26]修改 


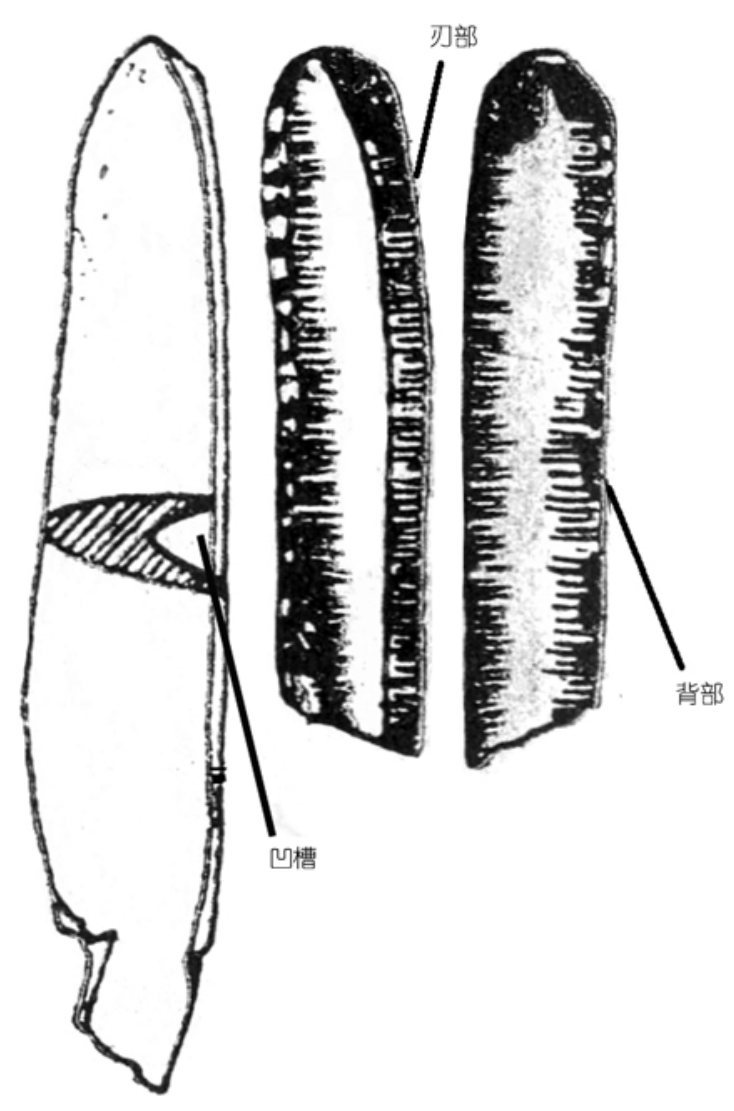

图 1 骨柄石刃刀

引自文献[24]图 3 10

处为器身中部, 深 $3.8 \mathrm{~mm}$, 残凹槽与骨柄器身等长. 石刃以细石叶为毛坏, 燧石, 青灰色, 长 $51 \mathrm{~mm}$, 宽 $10.5 \mathrm{~mm}$, 厚 $1.7 \mathrm{~mm}$, 重 $1.8 \mathrm{~g}$, 长宽之比为 4.86 , 唇 形台面, 打击泡比较突出, 有半雉体, 两侧边平行, 镶 嵌于刀柄凹槽内的细石叶部位采用压制法进行两面加 工, 疤痕较大. 刃缘部位锋利, 有微破损疮痕. 据肉眼 观察, 刃缘部位有一层光泽覆盖其上, 背缘处则无.

由于样品在 20 世纪 80 年代中期已经发掘出土, 其周围的土壤环境已不可知. 为了排除后期污染的 可能, 我们在做环扫观察时以台面向下、背面面对观 察者进行定位和标记, 对同一件样本不同部位进行 对比分析, 即区分刃部和非刃部、刃缘和背部以及同 一部位的物质覆盖部分和未覆盖部分.

实验在清华大学摩擦学实验室进行, 采用的是 荷兰 FEI 生产的 Quanta $200 \mathrm{FEG}$ 环境电子扫描显微 镜, 该仪器带有 $X$ 射线能谱仪, 采用低真空模式, 分 辨率在 $3 \mathrm{kV}<3.5 \mathrm{~nm}$ 时, 放大倍数为 $12 \sim 1000000$. 该
实验条件为电压 $15.0 \mathrm{kV}$, 环境压力 $80.0 \mathrm{~Pa}$, 使用了 低真空和背散射模式, 配合 $\mathrm{X}$ 射线能谱仪进行分析.

\section{2 环境扫描电镜观察结果}

\section{1 刃缘部位}

首先将刃缘部位放大至 500 倍进行观察, 刃缘中 部有较为密集且分布基本均匀的附着物(图 2(a), (b)). 附着物结构松散, 紧靠刃缘部位, 有与刃缘斜交的擦 痕两条. 进一步放大至 2500 倍, 刃缘微疤凹处亦有 同样的物质, 且附着较紧密(图 2(b)). 进一步放大至 9000 倍, 擦痕更加清晰, 残留物呈絮状聚集(图 2(c)). 在刃部选择 4 处进行了能谱分析 (图 2(c), (d)), 编号分 别为 $1,3,4,5$. 为了确定附着物是否为后期污染所致 (假设污染均一), 对以上 4 点做了作了 $\mathrm{EDX}$ 射线能谱 分析, 结果如图 3 所示.

标 1 点能谱分析表明该处物质基本元素为 $\mathrm{Si}, \mathrm{O}$, $\mathrm{C} ; \mathrm{Ca}$ 和 $\mathrm{Al}$ 的含量非常低(图 3(a)), 说明该样品基本 没有受到污染，可作进一步的深入分析. 标 3 点残留 物成分有 $\mathrm{Si}, \mathrm{O}, \mathrm{C}, \mathrm{Ca}, \mathrm{P}$ 等, 其中 $\mathrm{Ca}, \mathrm{P}, \mathrm{O}$ 的含量非常 的高. 标 4,5 点物质含有 $\mathrm{C}, \mathrm{O}, \mathrm{Si}, \mathrm{P}, \mathrm{Ca}$ 和少量的 $\mathrm{Na}$ 等物质成分, $\mathrm{Ca}, \mathrm{P}, \mathrm{O}$ 的含量也是非常的高; 这些元 素的可能组合为二氧化硅、磷酸钙以及碳氧化合物 等.

将刃缘局部放大至 2500 倍, 在其刃部边缘发现 有残留物, 其表面结构特征与其附着部位的物质形 态迥然不同(图 4(a)).X 射线能谱分析表明此物质高 $\mathrm{C}, \mathrm{O}$ 和 $\mathrm{Si}$, 含少量的 $\mathrm{Na}, \mathrm{Mg}, \mathrm{Al}, \mathrm{S}, \mathrm{Cl}, \mathrm{Ka}, \mathrm{Ca}, \mathrm{Fe}$ 等 物质, 其可能物质组合为碳氧化合物、二氧化硅及少 量的盐(图 4(b)), 并在此处附着物下发现了与刃部基 本斜交的两条擦痕.

\section{2 背缘部位}

首先对背缘放大至 100 倍进行面扫, 在很多破损 疤内均发现有附着物, 然后在靠近边缘部位逐步放大 倍率进行观察(图 5(a), (b)), 不同的放大倍率对物质 表面形态的认识是不一致的. 200 倍时, 该物质在一 定范围内片状连续分布; 1600 倍时, 该物质为粘连在 一起, 部并形成较为平滑的光面(图 5(b)). 能谱分析 表明其主要物质成分为 $\mathrm{C}, \mathrm{O}, \mathrm{Si}, \mathrm{Ca}, \mathrm{Mg}$ 等物质, 推 测其组合可能为有机物(碳氧化合物)、二氧化硅、 

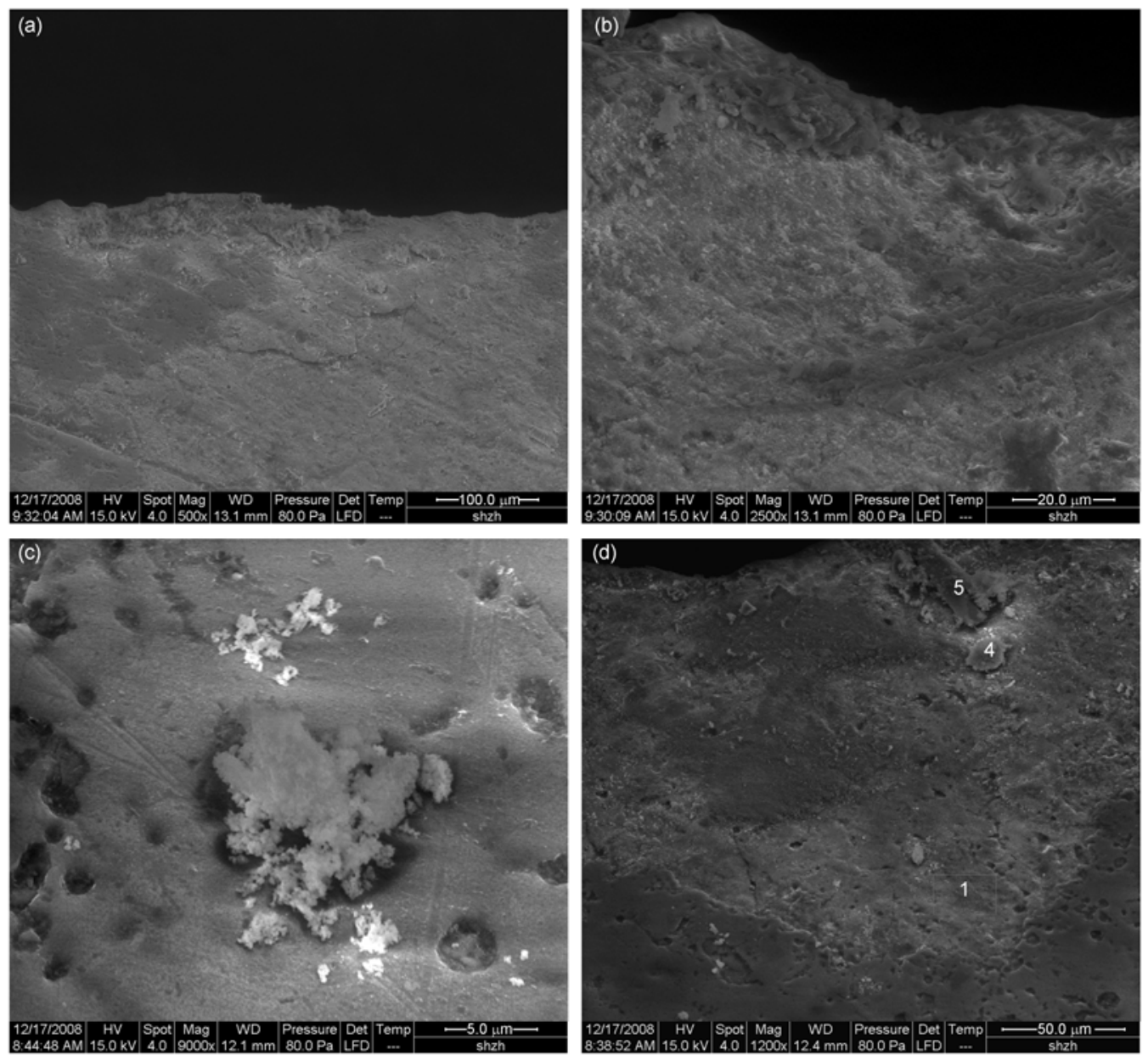

图 2 刃缘部位的微痕及残留物

(a) 斜向擦痕及残留物(500x); (b) 微疮内的残留物(2500x); (c) 残留物和擦痕及标 3 点 EDX 分析位置示意图(9000x); (d) 标 1, 4 和 5 点的 EDX 能谱分析位置示意图(1200x). 图像均为 SEI

碳酸钲、碳酸美等物质. 由于残留物层过薄, 扫描易 穿透, 故二氧化硅可能来自燧石, 所以推断其主要物 质成分为碳氧化合物 (图 5(e)).

放大至 11000 倍, 并添加接收背散射电子信号的 闪㷧计数器进行观察, 发现在该碳氧化合物内部有 均匀分布的较大原子序数的物质(图 5(c), (d)). 在图 5(d)标 3, 4 点做了能谱分析, 结果如图 5(f) 和 $(\mathrm{g})$, 均 含有 C, O, Ba, Zn, Mg, Si, S, Ka, Ca 等, 且 $\mathrm{Ba}, \mathrm{Zn}$ 含 量均较高, 故推测其主要成分为碳氧化合物、二氧化 硅、钡盐、镁盐、钙盐等物质.

\section{3 讨论}

图 3(b) (d)的分析结果表明, 刃缘部位残留物的
磷酸钙 $\mathrm{Ca}, \mathrm{P}$ 含量比较高, 而 $\mathrm{Ca}$ 和 $\mathrm{P}$ 是骨骼的主要组 成微量元素, 推测它们主要为骨质遗存的残留, 所以 该件骨柄石刀刀主要是处理肉类的工具, 并在使用 期间与骨质发生接触所致. 由于其尚保留了锋利的 刃缘 ${ }^{[27,28]}$, 推测其使用时间可能并不长. 废弃的主要 原因可能与骨柄部折断有关. 在刃缘部位发现的条 痕与 Olympics 金相显微镜在 200 倍时(图 2(a) (c))观 察得到条痕方向基本一致, 与刃缘斜交, 基本呈 $60 \sim 70^{\circ}$ 的角, 说明该器物在使用期间以向下斜切的 方向做运动.

刃部标 6 点(图 4(a)) 和背部 (图 5(b)) 附着物质的 微形貌截然不同. 其能谱分析(图 4(b), 图 5(e))表明, 残留物均为高碳、氧含量的有机物, 但它们组成元 

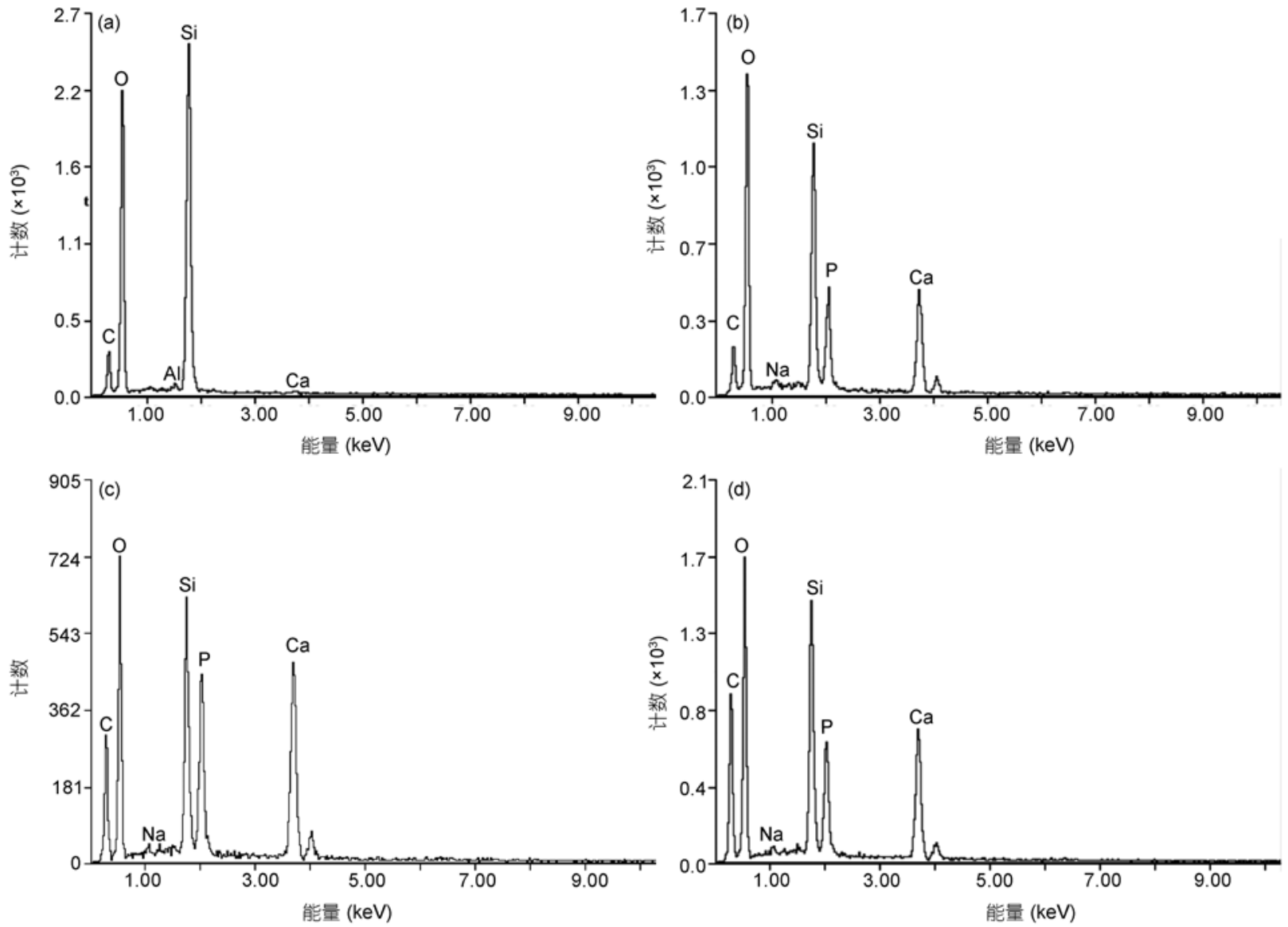

图 3 残留物的 EDX 分析

(a) (d) 分别为图 2(c)中标 3 点和图 2(d)中标 1, 4 和 5 点的 EDX 分析图谱. 纵坐标是测量时间内总的计数值; 在相同的测量时间内, 计数值与 元素含量相关; 横坐标为特征 X 射线所对应的能量. 下同
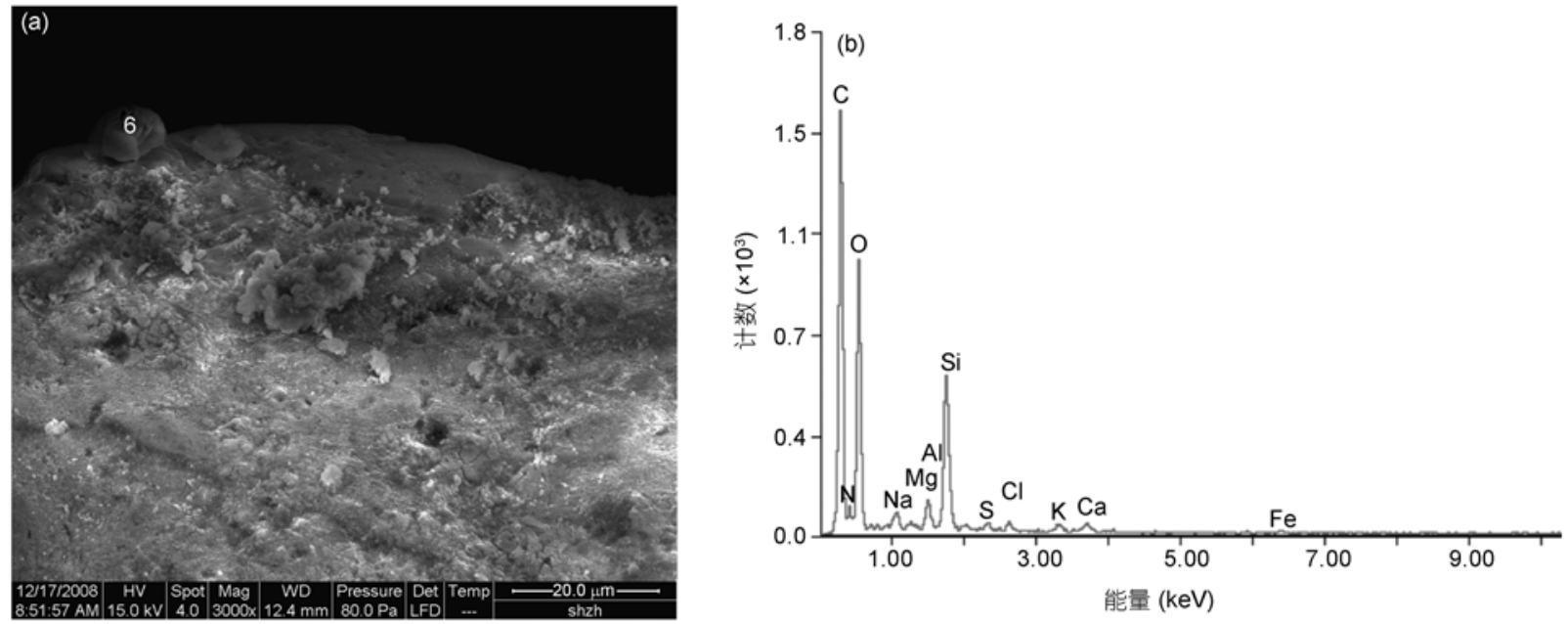

图 4 刃缘部位残留物的微形貌及其 EDX 分析

(a) 残留物的形态及表 6 点位置示意图(3000x, SEI); (b) (a)中标 6 点的 X 射线物质能谱分析图 

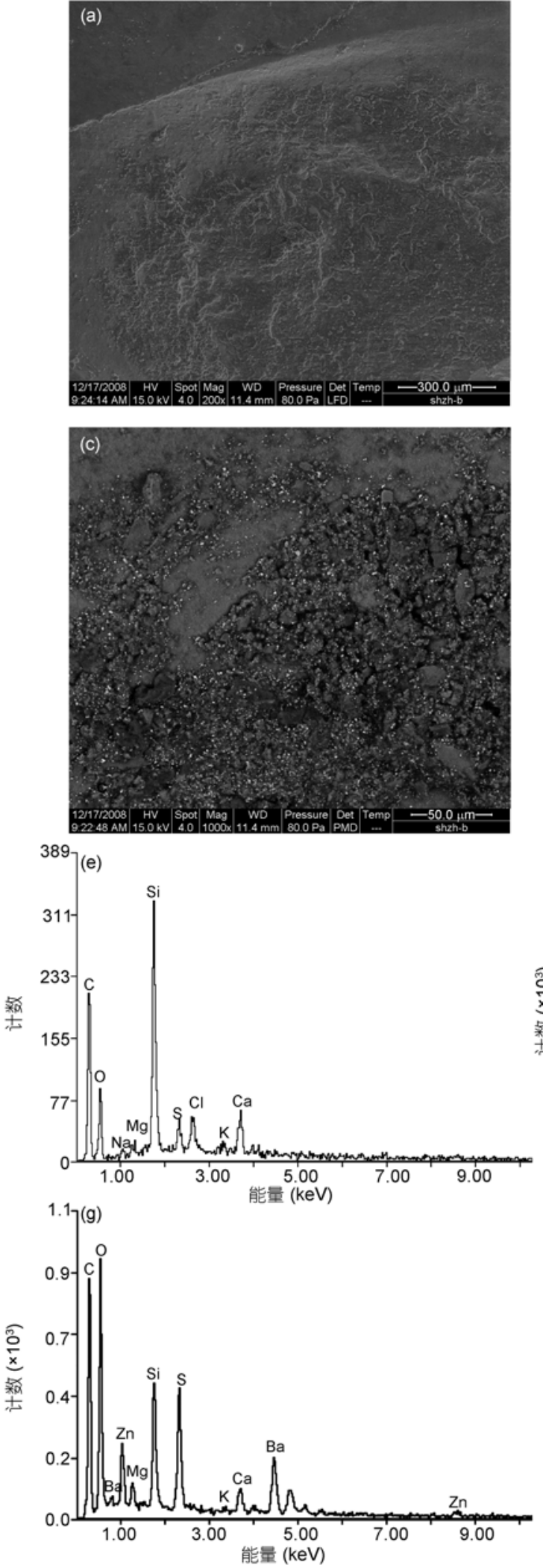
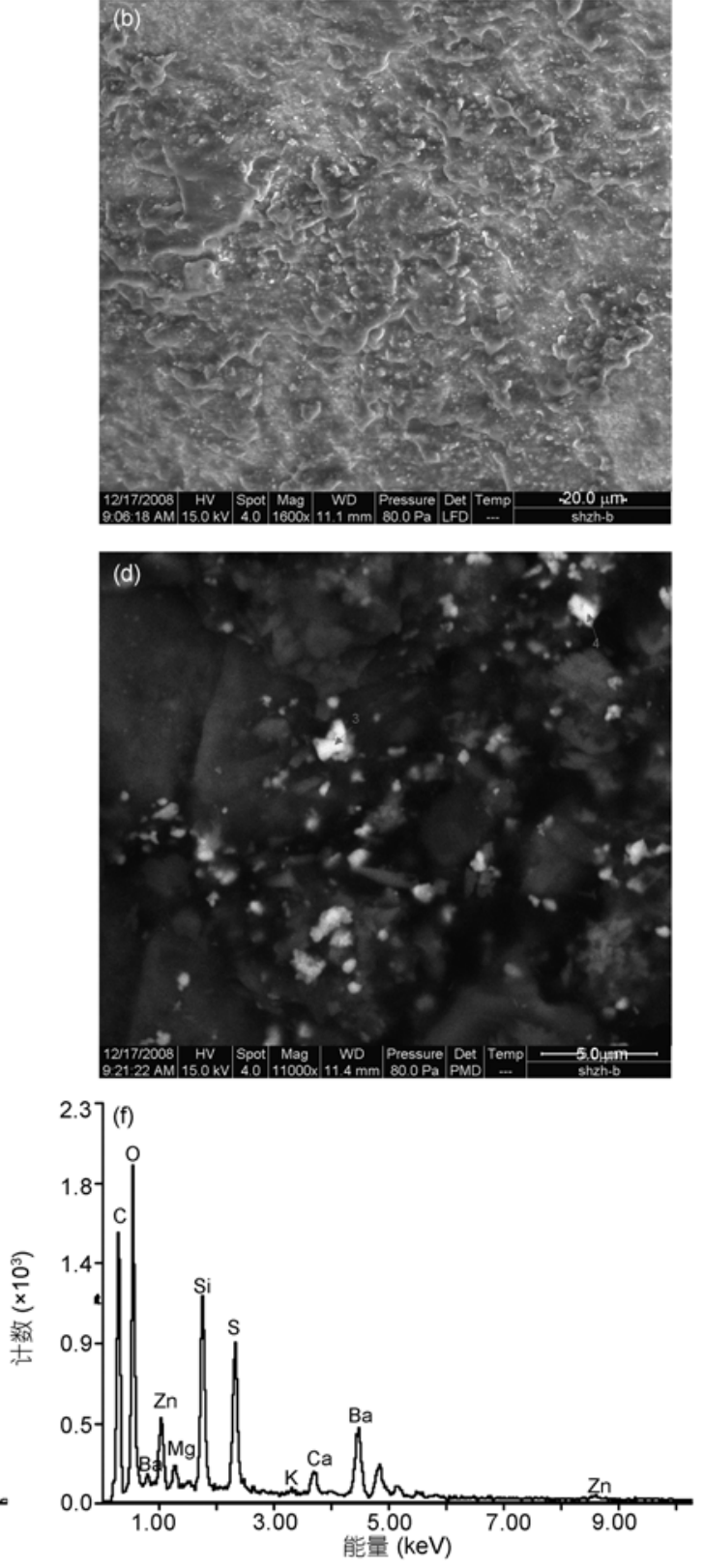

图 5 背缘部位的微痕、残留物微形貌及残留物的 EDX 分析

(a) 破损疤内残留物附着状态 $(200 \times)$; (b) 破损疤内残留物 的微形貌 $(1600 \times)$; (c) 残留物中较大原子序数的物质 (1000×, BEI); (d) 较大原子序数物质 X 射线能谱分析位置 图(11000x, BEI); (e)和 (b)中标 1 点 X 射线能谱分析图; (f)和 (d)中标 3 点 $X$ 射线能谱分析图; (g)和(d)中表 4 点 $X$ 射线能 谱分析图 
素存在明显的差异, 如图 4(b)能谱显示为高 $\mathrm{C}, \mathrm{O}$ 和 $\mathrm{Si}$, 含少量的 $\mathrm{N}, \mathrm{Na}, \mathrm{Mg}, \mathrm{Al}, \mathrm{S}, \mathrm{Cl}, \mathrm{Ka}, \mathrm{Ca}, \mathrm{Fe}$ 等元素, 微量 元素种类较多; 图 5(e)能谱显示为高 $\mathrm{C}, \mathrm{O}, \mathrm{Si}$ 及 $\mathrm{Ca}$, $\mathrm{Mg}$ 等元素, 其微量元素种类较少. 推测其组合为有机 物(碳氧化合物)、二氧化硅、钲镁类盐等. 据图 5(b), 残 留物的微形貌较平, 片状分布, 推测其为有机粘合胶.

图 5(a) (g)表明, 上宅文化的居民可能用有机胶 来固定细石叶. 该骨柄凹槽呈 V 字形(如图 1), 且凹 槽较浅. 为了固定, 除对细石叶进行修整使嵌入的骨 柄部分变得圆钝以利于结合紧密, 还需使用胶对其 进行粘合 ${ }^{[27]}$. 至于上宅古居民使用何种有机胶固定 细石叶, 尚需要进一步分析.

据图 5(e) (g), 在背散射图像(图 5(c), (d)) 中的高 亮点为钡盐、锌盐、镁盐、钻盐. 从图 5(c), (d)上可 以看出, 胶体中的添加物分布均匀, 颗粒较小, 约为 $1 \mu \mathrm{m}$ 左右. 由此可以得知这些添加物可能为人工有 意䚄入, 其作用尚不清楚. 但硫酸钡在现代常被用来 作为胶、颜料、橡胶中的填料, 用以减少胶结剂固化
后体积收缩率.

\section{4 结论与问题}

根据环境扫描电镜的观察结果, 可以得到以下 结论：1）上宅遗址出土的骨柄石刀刀是处理兽肉类 的工具；2）上宅遗址的史前居民对胶的性质有了一 定程度的了解, 如有意识在胶中掺入一些矿物如钡 盐. 在胶中幞合矿物质尚需要该遗址和其他遗址同 类资料的支持. 由于上述认识是据一个遗址中的一 件器物, 结论的局限性是比较大的, 故需要加大样本 数量进行深入的分析, 以期获得比较客观、全面的认 识.

本文表明, 发展成熟的环境扫描电镜技术非常 适宜于对导电性能不好或不导电的考古样品所携带 的信息进行深入的发掘, 为一些长期困扰史前学术 界的问题提供了一个新的解决途径, 如石器的微痕分 析.

致谢感谢北京大学黄蕴平教授、清华大学摩擦学国家重点学科实验室杨文言老师、中国科学院地理科学与资源研 究所杨晓燕博士、北京市平谷区上宅遗址陈列馆贾福胜先生、刘风英女士及Brown 女士对我们工作的支持 和帮助，感谢审稿人提出的修改意见.

\section{参考文献}

1 陈胜前. 细石叶工艺产品废弃的文化过程研究. 人类学学报, 2008, 27: 112-122

2 沈辰. 细石器工艺、细石器传统及山东细石器研究的初步认识. 见: 邓聪, 陈星灿, 编. 桃李成蹊集一一庆祝安志敏先生八十寿辰. 香港: 香港中文大学中国考古艺术研究中心, 2004. 45-56

3 安志敏. 中国细石器的一百年. 考古, 2000, (5): 45-56

4 安志敏. 海拉尔的中石器遗存一一兼论细石器的起源和传统, 考古学报, 1978, (3): 289-316

5 陈淳. 东亚和北美细石叶遗存的古环境, 第四纪研究, 1994, 24: 369-377

6 Harlan J R. Wild grass seed harvesting in the Sahara and sub-Sahara of Africa. In: Harris D R, Hillman G C, eds. Foraging and Farming: The Evolution of Plant Pxploitation. London: Unwin Hyman, 1989. 79-98

7 Unger-Hamilton R. The epipaleolithic southern Levant and the origins of plant cultivation. Curr Anthropol, 1989, 30: 88-103

8 Lü T L D. Some botanical characteristics of green foxtail (Setaria viridis) and harvesting experiments on the grass. Antiquity, 1998, 72: 902 $-907$

9 Lü T L D. The occurrence of the cereal in China. Asian Perspective, 2006, 45: 129-158

10 Lü T L D. A green foxtail (Setaria viridis) cultivation experimental in the middle Yellow River Valley and some related Issues. Asian Perspective, 2002, 41: 1-14

11 王小庆. 赵宝沟遗址细石叶微痕观察. 见: 邓聪, 陈星灿, 编. 桃李成蹊集一一庆祝安志敏先生八十寿辰. 香港: 香港中文大学中国 考古艺术研究中心, 2004. 112-120

12 李士, 秦广雍. 现代实验技术在考古中的应用. 北京: 科学出版社, 1983.112-118

13 Anderson P. A testimony of prehistory tasks: Diagnostic residues on stone tool working edge. World Archaeol, 1980, 12: 181—194

14 Dobney K, Broth W Ü R. Dental calculus: its relevance to ancient diet and oral ecology. In: Cruwys E, Foley R A, eds. Teeth and Anthropology. Oxford: BAR International Series, 1986. 55-81 
15 Carnelli A L, Madella M, Theurillat J P, et al. Aluminum in the opal silica reticule of phytoliths: A new tool in palaeoecological studies. Am J Botany, 2002, 89: 346-351

16 Devos W, Moens L, Bohlen A V, et al. Ultra-microanalysis of inorganic pigments on painted objects by total reflection X-Ray Fluorescence analysis. Stud Conservation, 1995, 40: 153-162

17 José-Yacamán M, Rendón L, Arenas J, et al. Maya blue paint: An ancient nano-structured material. Science, 1996, 273: 223-225

18 Knutsson K. Patterns of tool use-scanning electron microscopy of experimental quartz tools. Uppsala: Societas Archaeoloica Upsaliensis. 1988. $21-38$

19 侯亚梅. 考古标本微磨痕初步研究. 人类学学报, 1992, 11: 354-361

20 陈良标, 洪涛. 古尸寄生虫卵的扫描电子显微镜观察. 中国医学科学院学报, 1981, (1): 67一68, 79

21 王进玉. 中国古代青金石颜料的电镜分析. 文物保护与考古科学, 1997, 9: 25-32

22 马清林. 甘肃玉门火烧沟遗址出土红-黄色混合颜料的再分析. 文物保护与考古科学, 2006, 18: 4-8

23 夏寅, 吴双成, 崔圣宽, 等. 山东危山西汉墓出土陶器彩绘颜料研究. 文物保护与考古科学, 2008, 20: 13-19

24 冯敏, 张敬国, 王荣, 等. 凌家滩古玉受沁过程分析. 文物保护与考古科学, 2005, 17: 22-26

25 北京市文物研究所北京市平谷县文物管理所上宅考古队. 北京平谷上宅新石器时代遗址发掘简报. 文物, 1989, (8): 1-8

26 中国社会科学院考古研究所编. 中国考古学碳十四年代数据集(1965 1991). 北京：文物出版社, 1991. 17-18

27 郡达. 细石叶间接打片实验暨骨梗刀使用的微痕观察. 硕士学位论文. 北京大学考古文博学院, 1993. 1-23

28 科诺布科娃 G F. 古代镰刀及其使用痕迹之模拟实验研究. 张良仁, 译. 中国社会科学院考古研究所, 编. 考古学的历史.理论·实践. 郑州: 中州古籍出版社, 1996. 236-254 